\title{
Selección e indicadores para la evaluación del desarrollo sostenible de un destino turístico. Aplicación al municipio de Gijón
}

\author{
Raquel Álvarez Díaz \\ Universidad de Oviedo \\ raquelad@outlook.com \\ Luis Valdés Peláez \\ Universidad de Oviedo \\ lvaldes@uniovi.es
}

Recibido: 23-11-2015

Aceptado: 03-03-2016

Keywords: sustainable; indicators; Gijon

\begin{abstract}
Selection and indicators for assessing sustainable development of a tourist destination. Application to the municipality of Gijón. This research is dedicated to, on the one hand, establish a set of indicator that will make possible the analysis of the sustainable tourism development of the several municipalities and, on the other hand, it is also dedicated to the implementation of the mentioned set to Gijon's case. Once the conceptual basis are set about the main matters discussed, sustainable tourism and the use of indicators as a measurement tool, the system will be suggested. The indicators system is based on specific bibliographic references. Having the system been stablished, it will be applied to Gijon, after having gathered all the numbers and information necessary. Analyzing the results, what stands out is that Gijon is already working in terms of sustainability but there is a lack of communication and information about what they are really doing and achieving. Throughout this paper, it has been highlighted the need of a sustainable tourism model in destinations and that for achieving this, is necessary to have a tool that provides information about the previous situation of the destination as well as its evolution.
\end{abstract}

Palabras clave: sostenible; indicadores; Gijón

\section{RESUMEN}

Este trabajo está dedicado, por un lado, a la propuesta de un sistema de indicadores para el análisis del desarrollo turístico sostenible de destinos municipales $\mathrm{y}$, por otro, a analizar la situación actual de Gijón en materia de sostenibilidad a través de la aplicación de dicho sistema. Una vez establecida una base conceptual en torno a los principales temas tratados, el turismo sostenible y la utilización de indicadores como herramienta de medición, se propone un sistema basado en determinadas referencias bibliográficas. Con el sistema de indicadores ya constituido, se procede a su aplicación tras la obtención de las cifras y datos correspondientes. Tras llevar a cabo la metodología descrita y observar los resultados obtenidos, se observa que, a grandes rasgos, Gijón es un municipio que ya realiza acciones en torno a la sostenibilidad turística y se puede observar una evolución hacia la misma. A pesar de ello, existe una gran falta de comunicación e información en torno a las acciones llevadas a cabo. En este trabajo se ha tratado de exponer en todo momento la necesidad de implantar un modelo turístico sostenible en los destinos y que, para ello, es necesario disponer de una herramienta de medición que permita conocer la situación previa del municipio así como su evolución. 


\section{INTRODUCCIÓN}

Este trabajo nace de la necesidad de lograr un turismo viable económicamente, soportable socialmente y respetuoso con el medio ambiente. Es decir, de alcanzar y fomentar un desarrollo turístico sostenible (CE, 2013).

El concepto de sostenibilidad en el sector turístico ha evolucionado desde su aparición, cuando sólo se contempla su dimensión medioambiental, hasta hoy en dia que se considera como una herramienta para el desarrollo sostenible de las comunidades y como medio para fomentar un crecimiento de la sociedad (OMT, 2011).

Con el fin de conseguir esta tipología de turismo, es necesario que el nivel de sostenibilidad que alcanzan los distintos territorios sea medible. Esto, en la actualidad, es posible pero extremadamente dificil. Se han sucedido diversos trabajos y estudios que tenían como objetivo la construcción de sistemas de indicadores que facilitaran dicha medición, pero a día de hoy, no se ha logrado un sistema global y único (Sánchez y Pulido, 2011).

La dificultad de la cuantificación reside, por un lado, en el hecho de que la sostenibilidad es un concepto todavía muy confuso y amplio, en su carácter transversal, en la necesidad de utilizar diferentes unidades de medida y en la relatividad de la medición, dado que en función de la referencia tomada, los resultados y conclusiones de los indicadores pueden variar (Sánchez y Pulido, 2011).

Con el fin de facilitar la medición de la sostenibilidad de los destinos turísticos, en este trabajo se va a proceder a la elaboración de un sistema de indicadores que permita verificar y analizar el desarrollo turístico sostenible de Gijón, segunda ciudad en obtener la certificación BiosphereResponsibleTourism y que dispone de un Plan de Acción de Turismo Sostenible. Por lo tanto, se trata de un destino con antecedentes en torno a la materia tratada.

\section{OBJETIVOS DEL PROYECTO}

El objetivo general que se busca lograr a través de este trabajo y, por lo tanto, la meta final del mismo, es doble. Se pretende, por un lado, conocer la situación actual del desarrollo turístico sostenible del destino Gijón y, por otro, formular un sistema de indicadores de sostenibilidad turistica que pueda ser extrapolado a otros destinos municipales de características similares. Para alcanzar esta meta, se han fijado los siguientes objetivos específicos:

- Realizar una conceptualización en torno al concepto de turismo sostenible.

- Efectuar una breve investigación acerca de los sistemas de indicadores y su utilización en materia de turismo.

- Realizar un análisis previo de la situación turística actual del municipio de Gijón.

- Elaborar un sistema de indicadores para poder cuantificar el desarrollo turístico sostenible de un destino municipal.

- Aplicar los indicadores al destino Gijón para el periodo de tiempo seleccionado.

- Realizar un diagnóstico de la situación en materia de turismo sostenible en Gijón.

\section{METODOLOGÍA Y PLAN DE TRABAJO}

Este trabajo implica una parte práctica, pero también una parte teórica en la que se van a abordar diversos conceptos necesarios para una mayor comprensión de la sostenibilidad en el sector turístico y la utilización de indicadores. La conceptualización estará compuesta, por lo tanto, de una aproximación al turismo sostenible, a los sistemas de indicadores como herramientas de medición y de un análisis de Gijón, cuyo desarrollo turístico sostenible se trata de examinar.

Una vez finalizada la revisión literaria, se procederá a la aplicación práctica. Para ello, primero se hará una selección de los indicadores, basada en las referencias 
bibliográficas incluidas en la parte teórica del trabajo. Una vez seleccionados, se procederá a su cuantificación a través de la recopilación de información, su sistematización, cálculo y posterior análisis de los resultados. Las principales fuentes estadísticas para esto último serán el Sistema de Información Turística de Asturias (SITA) e InvesMark, empresa encargada de realizar los últimos informes disponibles acerca del turismo en Gijón.

Con el objetivo de, no sólo conocer la situación actual, sino la evolución y desarrollo del turismo sostenible, el sistema de indicadores se aplicará al periodo de tiempo comprendido entre 1997 y 2014, fijado en relación a los datos disponibles. Finalmente, se terminará el trabajo con una serie de conclusiones y propuestas cuyo objetivo es el de servir de base para posibles acciones a llevar a cabo en el destino.

\section{ANÁLISIS Y RESULTADOS}

Para la elaboración del sistema de indicadores cuyo objetivo es la medición del desarrollo turístico sostenible de Gijón, se van a tener en cuenta, en primer lugar, los Criterios Globales de Turismo Sostenible que servirán como referencia para establecer los objetivos de los indicadores. Y, en segundo lugar, el Sistema Español de Indicadores Ambientales de Turismo y la Herramienta del Sistema Europeo de Indicadores Turísticos para Destinos Sostenibles.

El sistema propuesto en este trabajo se divide en cuatro grandes bloques, los cuales corresponden a las tres dimensiones de la sostenibilidad y un cuarto dedicado al análisis de la gestión sostenible del territorio. Se ha procedido a hacer esta división con el fin de poder valorar el desarrollo del territorio no sólo en términos generales sino también dependiendo de la dimensión a la que pertenece cada indicador. De esta forma, se podrá observar si existen grandes diferencias entre los tres componentes de la sostenibilidad y su evolución. Al mismo tiempo, se ha decidido dedicar un bloque en exclusividad a la gestión sostenible del destino para poder así analizar este aspecto de manera más exhaustiva.

Para la elección de los indicadores, se ha llevado a cabo una revisión bibliográfica tras la cual se ha decidido considerar los indicadores propuestos por el Ministerio de Medio Ambiente y por la Comisión Europea, ambas herramientas mencionadas con anterioridad. Partiendo de este listado inicial, se han eliminado los que no es posible cuantificar por falta de datos.

Con el fin de poder analizar el desarrollo turístico sostenible y no únicamente la situación actual del territorio, se va a proceder a examinar la evolución de los indicadores a lo largo del tiempo. Para ello se van a tomar de referencia los informes territoriales de Gijón del SITA, los cuales están disponibles desde 1997 hasta 2012 y el informe de InvesMark correspondiente al 2013 y el avance de su informe de 2014.

Dada la amplitud del periodo 1997-2014 se ha optado por seleccionar sólo determinados años. Por ello, en primer lugar, se ha decidido reducir dicho periodo al comprendido entre 2005-2014 dado que a raíz de 2005 se empieza a utilizar una metodología prácticamente similar a la utilizada a día de hoy y, por lo tanto, por la simplificación que supone para la búsqueda y obtención de datos.

Una vez reducido el periodo, se ha optado por realizar un análisis de los datos de cada tres años, con el fin de seleccionar pocos años y así poder llevar a cabo un análisis más exhaustivo pero no demasiado extenso. En definitiva, se han elegido los años 2005, 2008, 2011 y 2014. trabajo:

A continuación se muestran los indicadores que forman el sistema propuesto en este 
Tabla 1. Sistema de indicadores propuesto: Gestión Sostenible del Destino

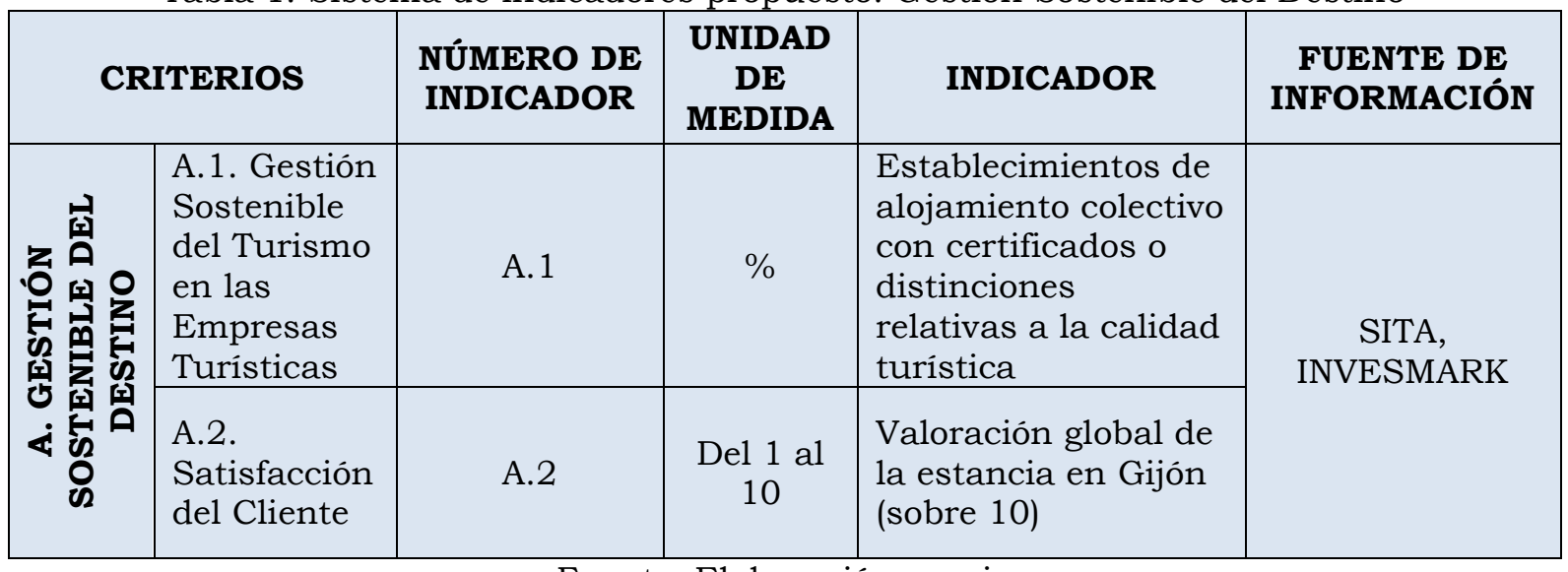

Fuente: Elaboración propia

Tabla 2. Sistema de indicadores propuesto: Dimensión Económica

\begin{tabular}{|c|c|c|c|c|c|}
\hline \multicolumn{2}{|c|}{ CRITERIOS } & \multirow{2}{*}{$\begin{array}{c}\text { NÚMERO DE } \\
\text { INDICADOR } \\
\text { B. } 1.1 \\
\end{array}$} & \multirow{2}{*}{$\begin{array}{c}\text { UNIDAD DE } \\
\text { MEDIDA } \\
\% \\
\end{array}$} & \multirow{2}{*}{\begin{tabular}{l}
\multicolumn{1}{c}{ INDICADOR } \\
Número de turistas \\
que pernoctan al mes
\end{tabular}} & \multirow{2}{*}{$\begin{array}{c}\begin{array}{c}\text { FUENTE DE } \\
\text { INFORMACIÓN }\end{array} \\
\text { EOH (INE) }\end{array}$} \\
\hline \multirow{10}{*}{ 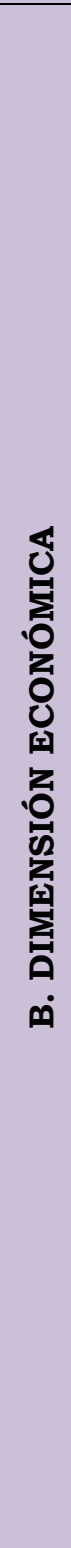 } & \multirow{5}{*}{$\begin{array}{l}\text { B.1. Flujo } \\
\text { Turistico } \\
\text { en el } \\
\text { Destino } \\
\text { (volumen } \\
\& \text { valor) }\end{array}$} & & & & \\
\hline & & B. 1.2 & $\%$ & $\begin{array}{l}\text { Aportación del } \\
\text { turismo al VAB }\end{array}$ & \multirow{5}{*}{$\begin{array}{c}\text { SITA, } \\
\text { INVESMARK }\end{array}$} \\
\hline & & B.1.3 & $\begin{array}{c}\text { Miles de } € / \\
\% \Delta\end{array}$ & $\begin{array}{l}\text { Gasto turístico total } \\
\text { anual }\end{array}$ & \\
\hline & & B. 1.4 & $€ / \% \Delta$ & $\begin{array}{l}\text { Gasto diario por } \\
\text { excursionista }\end{array}$ & \\
\hline & & B. 1.5 & $€ / \% \Delta$ & $\begin{array}{l}\text { Gasto diario por } \\
\text { turista (alojamiento, } \\
\text { alimentación y } \\
\text { bebidas, otros } \\
\text { servicios) }\end{array}$ & \\
\hline & \multirow{4}{*}{$\begin{array}{l}\text { B.2. } \\
\text { Resultados } \\
\text { de las } \\
\text { Empresas } \\
\text { Turísticas }\end{array}$} & B. 2.1 & Noches & $\begin{array}{l}\text { Duración media de la } \\
\text { estancia de un turista } \\
\text { (noches) }\end{array}$ & \\
\hline & & B.2.2 & $\%$ & $\begin{array}{l}\text { Grado de ocupación } \\
\text { por plazas en } \\
\text { alojamiento colectivo } \\
\text { (mensual y media } \\
\text { anual) }\end{array}$ & $\mathrm{EOH}$ (INE) \\
\hline & & B.2.3 & $€ / \% \Delta$ & $\begin{array}{l}\text { Evolución del } \\
\text { indicador de } \\
\text { rentabilidad ADR } \\
\text { (AverageDailyRevenue }\end{array}$ & \multirow{2}{*}{ INVESMARK } \\
\hline & & B. 2.4 & $€ / \% \Delta$ & $\begin{array}{l}\text { Evolución del } \\
\text { indicador de } \\
\text { rentabilidad RevPar } \\
\text { (Revenue Per } \\
\text { AvailableRoom) }\end{array}$ & \\
\hline & $\begin{array}{l}\text { B.3. } \\
\text { Cantidad } \\
\text { del Empleo }\end{array}$ & B.3. & $\begin{array}{c}\text { Trabajador/ } \\
\text { Plaza } \\
\text { disponible }\end{array}$ & $\begin{array}{l}\text { Empleo en servicios } \\
\text { de alojamiento según } \\
\text { plazas disponibles } \\
\text { (trabajadores } \\
\text { afiliados) }\end{array}$ & IET \\
\hline
\end{tabular}


Fuente: Elaboración propia

Tabla 3. Sistema de indicadores propuesto: Dimensión Social/Cultural

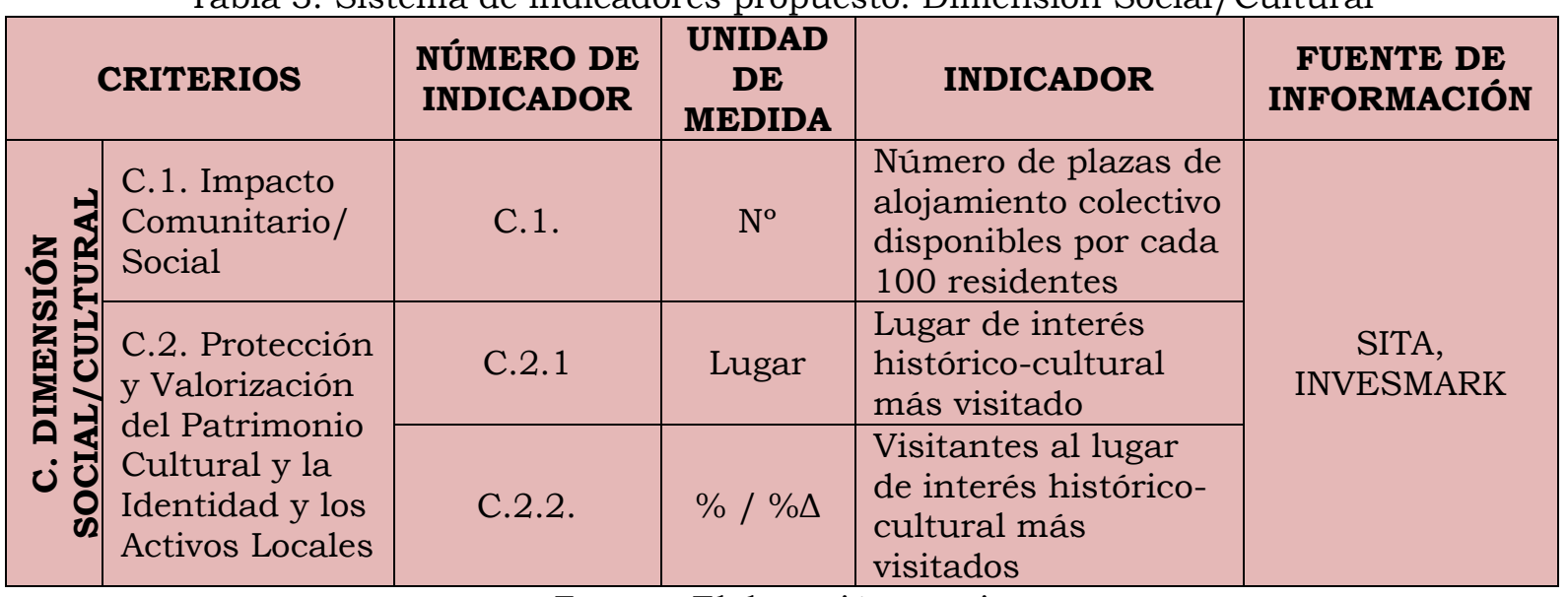

Fuente: Elaboración propia

Tabla 4. Sistema de indicadores propuesto: Dimensión Medioambiental

\begin{tabular}{|c|c|c|c|c|c|}
\hline \multicolumn{2}{|c|}{ CRITERIOS } & $\begin{array}{l}\text { NÚMERO DE } \\
\text { INDICADOR }\end{array}$ & $\begin{array}{l}\text { UNIDAD } \\
\text { DE } \\
\text { MEDIDA }\end{array}$ & INDICADOR & $\begin{array}{l}\text { FUENTE DE } \\
\text { INFORMACIÓN }\end{array}$ \\
\hline \multirow{7}{*}{ 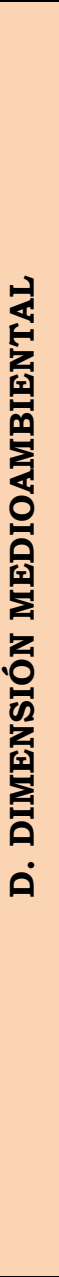 } & $\begin{array}{l}\text { D.1. } \\
\text { Gestión de } \\
\text { los } \\
\text { Residuos } \\
\end{array}$ & D. 1 . & $\%$ & $\begin{array}{l}\text { Establecimientos de } \\
\text { alojamiento colectivo que } \\
\text { cuentan con recogida selectiva } \\
\text { de basuras }\end{array}$ & \multirow{6}{*}{$\begin{array}{c}\text { SITA, } \\
\text { INVESMARK }\end{array}$} \\
\hline & \multirow{2}{*}{$\begin{array}{l}\text { D. } 2 . \\
\text { Gestión } \\
\text { del } \\
\text { Agua/Agu } \\
\text { as } \\
\text { residuales }\end{array}$} & D.2.1. & $\%$ & $\begin{array}{l}\text { Establecimientos de } \\
\text { alojamiento colectivo que } \\
\text { disponen de sistemas de } \\
\text { depuración y reutilización de } \\
\text { aguas residuales }\end{array}$ & \\
\hline & & D.2.2. & $\%$ & $\begin{array}{l}\text { Establecimientos de } \\
\text { alojamiento colectivo que } \\
\text { cuentan con sistemas de } \\
\text { limitación de consumo de agua }\end{array}$ & \\
\hline & \multirow{3}{*}{$\begin{array}{l}\text { D.3. } \\
\text { Gestión de } \\
\text { la Luz }\end{array}$} & D.3.1. & $\%$ & $\begin{array}{l}\text { Establecimientos de } \\
\text { alojamiento colectivo que } \\
\text { utilizan bombillas de bajo } \\
\text { consumo e interruptores de } \\
\text { presencia }\end{array}$ & \\
\hline & & D.3.2. & $\%$ & $\begin{array}{l}\text { Establecimientos de } \\
\text { alojamiento colectivo que } \\
\text { cuentan con paneles solares }\end{array}$ & \\
\hline & & D.3.3. & $\%$ & $\begin{array}{l}\text { Establecimientos de } \\
\text { alojamiento colectivo que } \\
\text { disponen de sistemas de } \\
\text { limitación de uso de energía }\end{array}$ & \\
\hline & $\begin{array}{l}\text { D. } 4 . \\
\text { Calidad de } \\
\text { las Aguas } \\
\text { de Baño }\end{array}$ & D.4. & $\begin{array}{l}\text { Muy } \\
\text { buena/ } \\
\text { Buena/ } \\
\text { No Apta } \\
\text { para el } \\
\text { baño }\end{array}$ & $\begin{array}{l}\text { Calidad de las aguas de baño } \\
\text { de las playas }\end{array}$ & $\begin{array}{l}\text { Consejería de } \\
\text { Sanidad } \\
\text { (Gobierno del } \\
\text { Principado de } \\
\text { Asturias) }\end{array}$ \\
\hline
\end{tabular}

Fuente: Elaboración propia 


\section{Gestión Sostenible del Destino}

Gracias a los indicadores que se han cuantificado, se puede comprobar que los establecimientos de alojamiento colectivo con certificados o distinciones relativas a la calidad turística han aumentado con el paso de los años. Por lo tanto, se puede comprobar cierta toma de conciencia por parte de estas empresas.

Otro indicador nos muestra la valoración global de la estancia en Gijón por parte de sus visitantes, que en el periodo analizado ha ido en aumento. Se puede afirmar, entonces, que en torno a la Gestión Sostenible del Destino los datos son positivos habiendo aumentado tanto las empresas con certificaciones relativas a la calidad y/o sostenibilidad, como la valoración del destino por parte de los visitantes.

\section{Dimensión económica}

La dimensión económica es una para las que más indicadores se han seleccionado, dado que se tratan datos que, en general, han sido recogidos desde el comienzo de la emisión de los informes sobre el turismo en Gijón por el SIITA en 1997.

La aportación del turismo al VAB se ha visto afectada de manera negativa habiéndose reducido, su causa se puede observar en el indicador que cuantifica el gasto turístico total anual, que también se ha visto reducido. A su vez, para justificar el descenso del gasto turístico total anual, se han de tener en cuenta los gastos diarios de excursionistas y turistas. Estos dos datos presentan hasta la fecha una tendencia a la baja.

En cuanto a los resultados de las empresas turísticas, la estancia media de los turistas ha disminuido. Los turistas se decantan así por realizar un mayor número de viajes a lo largo del año pero de menor duración cada uno. La media del grado de ocupación por plazas de las empresas de alojamiento colectivo, no se ha visto afectado de manera considerable durante el periodo 2005-2011. Aunque de 2011 a 2013 la variación ha sido mayor, detectándose un ligero descenso en prácticamente todos los meses.

El ADR y RevPar muestran un descenso de la rentabilidad de las empresas, aún y no habiendo sufrido descensos notables en número de viajeros ni pernoctaciones, atribuible a la disminución ya analizada del gasto turístico.

Por otra parte, se puede observar que a lo largo de los años analizados, el número de trabajadores afiliados en los sectores correspondientes a la hostelería y agencia de viajes, se ha visto aumentado. Puede que en aras de prestar una atención más personalizada y cercana a los visitantes al destino tratando así de mejorar la propia valoración de los visitantes del municipio.

\section{Dimensión social/cultural}

En el periodo analizado, el número de plazas de alojamiento turístico disponibles por cada 100 habitantes, ha aumentado ligeramente hasta el 2011. Por lo que el impacto sobre los habitantes es mínimo, no existiendo un crecimiento desmesurado ni descontrolado de la oferta.

El lugar de interés histórico-cultural más visitado desde 2005 ha sido el mismo, el Casco Antiguo - Cimadevilla. El resultado de este indicador sería interesante tenerlo en cuenta con el fin de plantearse, o no, un refuerzo en el mantenimiento y protección del lugar mencionado. Por otro lado, también podría tenerse en cuenta para llevar acciones de fomento de la cultura y patrimonio propio del destino en dicho lugar.

Además, el porcentaje de visitantes a lugares caracterizados por su esencia histórica y/o cultural, ha ido en aumento, aunque en el último año ha descendido ligeramente. Por lo que recalcar una vez más que es importante que se proteja el patrimonio a la vez que se valora y fomenta. Debería tenerse muy en cuenta este aumento sobre todo porque en comparación con los visitantes de 2005, los actuales suponen casi el doble, y este rápido crecimiento ha podido derivar en escasos o incluso inexistentes medidas de protección del patrimonio del destino.

Aunque estos indicadores pueden parecer no concluyentes, se ha decidido incluirlos tras observar que no ha habido variación en el lugar más visitado. Ya que en caso de haber una gran variación, no se podrían sacar conclusiones claras pero al no variar, resulta 
relevante al implicar cierto análisis en torno a la promoción que se realiza de este lugar y el posible deterioro que esté sufriendo.

\section{Dimensión medioambiental}

Los establecimientos de alojamiento que cuentan con recogida selectiva de residuos han aumentado con el paso de los años, pero la última cifra muestra un marcado descenso. Los datos del resto de años fueron extraídos de informes del SITA mientras que este último corresponde al informe de InvesMark. Puede deberse, por lo tanto, a un cambio en la cuantificación o por el muestreo, ya que este cambio no parece lógico al no ser muy comprensible eliminar la recogida selectiva de basura de un establecimiento de alojamiento, aunque no se descarta.

Para el análisis de la gestión del agua/aguas residuales, se han calculado indicadores relacionados con los establecimientos de alojamiento colectivo, ya que sólo se dispone de información relativa a estas empresas turísticas. De este tipo de empresas, en los informes de turismo de Gijón se dispone de la información acerca de aquellas que cuentan con sistemas de limitación de consumo de agua. En 2008, respecto a 2005, las empresas que disponian de dichos sistemas disminuyeron en ambos casos. Pero en 2011, aumentaron llegando incluso a doblarse en número si se compara con 2005. Los alojamientos colectivos con sistemas de depuración y reutilización de aguas residuales son menores, puede que por la mayor dificultad de instalar dichos sistemas.

Para la gestión de la luz, todos los indicadores muestran una tendencia al alza, habiendo crecido el número de establecimientos de alojamiento colectivo que utilizan bombillas de bajo consumo, los que cuentan con paneles solares y los que disponen de sistemas de limitación de uso de energía. Queda así patente que cada vez son más las empresas de esta tipología que muestran una gestión de la luz más respetuosa con el medioambiente.

Por último, la calidad de las aguas de baño desde 2011 que es el primer año del que se dispone del dato, ha sido y es muy buena para todas las playas de Gijón sin excepción.

\section{CONCLUSIONES E IMPLICACIONES}

A lo largo de los últimos años, se ha podido demostrar que es necesario implantar un modelo de turismo sostenible en los destinos con el fin de preservar sus caracteristicas a lo largo del tiempo y que así puedan ser disfrutadas tanto por las generaciones actuales como por las futuras. Pero con el objetivo de implantar medidas para alcanzar este tipo de turismo, es necesario realizar un análisis previo del territorio para conocer su situación previa y hacer un seguimiento del desarrollo del mismo.

Implantar un turismo sostenible en Gijón debería ser primordial para las administraciones y empresas del municipio, dado que cada vez existe mayor concienciación por parte de instituciones y de la sociedad en general acerca de la preservación del territorio y patrimonio. Logrando un modelo sostenible, se consigue minimizar los impactos negativos de la actividad turística al mismo tiempo que se maximizan los beneficios. A su vez, se logra conservar la esencia del destino evitando la destrucción de su valor.

Desconocer la situación actual de un territorio en materia de sostenibilidad, imposibilita el avance hacia un modelo respetuoso medioambiental, social y económicamente. Es por ello que es necesaria una herramienta que permita un desarrollo constante y la mejora continua, como puede ser un sistema de indicadores. Para que esta herramienta sea útil y creíble, es necesario que sus fuentes de información sean identificadas explícitamente y los datos sean verificables de manera objetiva. Ambos requisitos se han tratado de cumplir en todo momento a lo largo de este trabajo.

Por otra parte, la lista de indicadores debe ser reducida con el fin de que la información se maximice y los costes se minimicen. Esta condición también se ha cumplido, aunque hay indicadores que no se han podido incluir por falta de información y que podrian haber resultado muy relevantes para el análisis de Gijón. 
A la hora de elaborar el sistema de indicadores, las mayores limitaciones se han dado en la recopilación de información sobre la gestión sostenible eficaz y la dimensión social/cultural, ambos apartados son los que menos indicadores poseen siendo su evaluación, por lo tanto, escasa. Dentro del primer apartado, correspondiente a la Gestión Sostenible del Destino, hubiera sido relevante incluir indicadores que cuantificaran la participación de los habitantes en le gestión del destino. Así como la consideración de otras empresas turísticas y no únicamente las de alojamiento. En cuanto a la dimensión social/cultual, la otra más afectada por las limitaciones de este trabajo, se hubieran incluido si hubiera sido posible indicadores referentes a la calidad del empleo turístico.

Pero la dimensión económica y la medioambiental, aunque dispongan de un mayor número de indicadores, no han estado exentas de los efectos de la falta de información. En el primero de ellos, el económico, no se ha podido incluir cuantificación alguna de la igualdad de género y la igualdad/accesibilidad en los diferentes establecimientos, medios de transporte y atracciones turisticas del municipio. Además de la opinión de los propios habitantes sobre cómo les afecta el turismo. En la dimensión medioambiental, no se ha podido acceder a cifras acerca de la generación y recogida de residuos totales (no únicamente los correspondientes a la recogida selectiva), de los consumos de los turistas de energía y agua para poder realizar una comparación con los habitantes, ni sobre la protección del paisaje y la biodiversidad.

Tras el análisis realizado a Gijón para determinar su desarrollo turístico sostenible a través de la aplicación del sistema de indicadores previamente propuesto, son varias las conclusiones a las que se ha podido llegar.

El análisis nos muestra concienciación por parte de las empresas, las cuales cada vez son más con certificaciones de calidad y/o medio ambiente y con sistemas de depuración y reutilización de aguas residuales, recogida selectiva de basuras, limitación de consumo de agua y de energía, así como aquellas que usan bombillas de bajo consumo e interruptores de presencia y paneles solares. Estas empresas contribuyen así a lograr la eficiencia de los recursos, a la pureza medioambiental y al uso óptimo de los recursos medioambientales. Además, esta concienciación muestra una predisposición empresarial que resulta interesante a la hora de plantear iniciativas o proyectos en materia de sostenibilidad, ya que se puede esperar cierta implicación por parte del sector privado.

El incremento de la valoración global de la estancia en Gijón es importante para el desarrollo turístico sostenible ya que se ha de logar un alto grado de satisfacción por parte de los visitantes y Gijón, en ese sentido, ha logrado el objetivo mejorando cada año dicho dato.

De la distribución mensual de las pernoctaciones, resalta la alta estacionalidad a la que está sometido el municipio y que, a lo largo del periodo, no ha disminuido. Convendria tenerlo presente a la hora de promover el turismo con el fin de fomentar los viajes de congresos y negocios, por ejemplo, para conseguir disipar dicha estacionalidad.

En términos económicos, el gasto diario por turista y excursionista ha disminuido produciendo un efecto dominó que ha derivado en la disminución también del gasto turístico total y de la aportación al VAB del turismo. Poniendo en peligro la viabilidad económica a largo plazo. Ha disminuido también la estancia media y la rentabilidad de las empresas a la vez que ha crecido el empleo. El sector turístico debería velar por si rentabilidad económica, aunque se ha de destacar el beneficio social reportado: la creación de empleo.

El incremento de las plazas disponibles por alojamiento ha incrementado aunque ligeramente. Por lo que el impacto producido sobre la población local puede suponerse que es mínimo, no habiendo un crecimiento masivo de la oferta.

El número de visitantes de los lugares de interés histórico-cultural ha incrementado de manera considerable, además de haberse mantenido en el tiempo el lugar más visitado. Debe tenerse en cuenta para poder garantizar la riqueza cultural y que ésta no se vea afectada de manera negativa. Si se comparan los años 2005 y 2013, estos visitantes han llegado casi a doblar su número. Un incremento tan notorio puede requerir medidas 
preventivas con el fin de no superar la capacidad de carga de estos lugares y no destruir sus características esenciales.

Tras el análisis realizado se puede afirmar que Gijón, en términos generales, avanza en el camino adecuado hacia un turismo sostenible. Aunque, a día de hoy, todavía queda mucho por hacer. Son muchas las acciones que parecen llevarse a cabo pero existe una falta de comunicación que dificulta el poder profundizar. Si se mejorara se lograría además una mayor participación por parte de todos los agentes implicados en el desarrollo turístico sostenible del destino.

Con ese objetivo, podría tenerse en cuenta la posibilidad de la creación de un informe paralelo al relativo al turismo en el municipio, incluyendo todos aquellos aspectos que contribuyen a la sostenibilidad del destino. Se ha podido observar, que con el paso de los años, cada vez se iban añadiendo más apartados en los informes relativos al turismo sostenible. Se ha llegado a tal punto, que en mi opinión, creo que sería viable la redacción de uno exclusivamente dedicado a esta materia.

En términos económicos, el destino no está bien encaminado hacia la sostenibilidad económica a largo plazo, ya que sus valores presentan tendencias a la baja. Aunque se ha de tener en cuenta la inestabilidad nacional actual, la cual afecta negativamente al país. La gestión sostenible del destino, aunque ha sido evaluada de manera muy leve, parece presentar una buena evolución hacia la sostenibilidad. Sobre la dimensión social/cultural, recalcar una vez más, la necesidad de preservar el patrimonio del destino sobre todo en aquellos lugares altamente frecuentados, a la vez que se fomenta la visita a aquellos que reciben menos visitas en busca de un equilibrio. Y, por último, acerca del medio ambiente, parece ser la dimensión que se encuentra en mejor situación en Gijón, existiendo una alta participación por parte de las empresas para lograr estos buenos resultados.

\section{REFERENCIAS BIBLIOGRÁFICAS}

Agencia de Ecologia Urbana de Barcelona (2010). Plan de Indicadores de Sostenibilidad Urbana de Vitoria-Gasteiz.

CE, (2013). Herramienta del Sistema Europeo de Indicadores Turisticos para Destinos Sostenibles.

Consejeria de Medio Ambiente, Ordenación de Territorio e Infraestructuras (2009). Análisis de la Huella Ecológica en el Principado de Asturias.

CMMAD, (1988). Nuestro futuro común. Alianza.

Dupeyras, A and N. MacCallum (2013). "Indicators for Measuring Competitiveness in Tourism: A Guidance Document”, OECD Tourism Papers, 2013/02, OECD.

GSTC, (2008). Global Sustainable Tourism Criteria. The Partnership for Global Sustainable Tourism Criteria.

GSTC, (2012). Criterios globales de turismo sostenible para destinos.

InvesMark, (2014). El turismo en Gijón 2013.

InvesMark, (2015). Avance el Turismo en Gijón 2014.

Ivars et al. (2001). Planificación y gestión del desarrollo turístico sostenible: propuestas para la creación de un sistema de indicadores. Documento de Trabajo Instituto Universitario de Geografia $\mathrm{n}^{\circ} 1$. Universidad de Alicante.

Ivars, J.A. (2003). Planificación turística de los espacios regionales en España,Madrid: Sintesis.

Ministerio de Medio Ambiente (2003). Sistema español de indicadores ambientales de turismo, Madrid. Ministerio de Medio Ambiente.

OCDE, (1997). Better understanding our cities. The rule of urban indicators. OCDE, Paris.

OMT, (1995). Lo que todo gestor turístico debe saber. Guía práctica para el desarrollo y uso de indicadores de turismo sostenible. Madrid.

OMT, (2011). Politicas y prácticas para el turismo mundial. Madrid.

OMT, (2013). Panorama OMT del turismo internacional, edición 2013.

OMT y PNUMA, (2005). Por un turismo más sostenible: Guía para responsables politicos. Madrid. 
ONU, (1992). Informe de la Conferencia de las Naciones Unidas sobre el Medio Ambiente y el Desarrollo.

Pulido Fernández, J., y Sánchez Rivero, M. (2011). Análisis dinámico de la sostenibilidad turística en España desde una perspectiva regional. Palma: Universidad de las Islas Baleares.

Rueda (1999). Modelos e indicadores para ciudades más sostenibles. Departament de MediAmbient de la Generalitat de Catalunya.

SITA (1997, 2005, 2008, 2011). El Turismo en Gijón. Disponibles en http://www.sita.org/

SITA (2015). Resultados Anuales Concejos 2004-2014. Disponible en http://www.sita.org/

ThielEllul, D. (2014). Medición y análisis de la sostenibilidad: Indicadores sintéticos a través de métodos multicriterio y su relación con el turismo en el litoral de Andalucía (Posgrado). Universidad de Málaga.

Valls, J. (2004). Gestión de destinos turísticos sostenibles. Madrid: Ediciones Gestión 2000.

\section{Páginas web consultadas}

AGICATUR, (s.f.). Asociación de Gijón para la Calidad Turística. Recuperado el 02 de Junio de 2015, de http://www.gijoncalidad.com/index.html

Ayuntamiento de Gijón, (s.f.). Ayuntamiento de Gijón. Recuperado el 15 de Abril de 2015, de http://www.gijon.info/page/12528-gijon-sostenible

Gobierno del Principado de Asturias, (2014). Consejeria de Sanidad. Calificación sanitaria de del agua de baño de las playas asturianas temporada 2011-2014. Recuperado el 15 de Mayo de 2015, dehttp://www.asturias.es/Asturias/playas/calificacion.htm

GSTC,. (s.f.). Global Sustainable Tourism Council. Recuperado el 28 Abril 2015, de https://www.gstcouncil.org/

IET (2015). Instituto de Estudios Turísticos. Recuperado el 20 de Mayo de 2015, de http://www.iet.tourspain.es/es-ES/Paginas/default.aspx

Empleo Turístico

INE (2015). Instituto Nacional de Estadística. Recuperado el 19 de Mayo de 2015, de http://www.ine.es/

Encuesta Ocupación Hotelera

INE (2014). Instituto Nacional de Estadística. Recuperado el 19 de Mayo de 2015, de http://www.ine.es/

$>\quad$ Estadística Padrón Continuo

$>\quad$ Encuesta de Población Activa

OMT, (s.f.). Organización Mundial del Turismo. Recuperado el 15 de Mayo de 2015, de http://www2.unwto.org/es

HOW TO CITE THIS ARTICLE IN BIBLIOGRAPHIE

Álvarez Díaz, R. y Valdés Peláez, L. (2016): "Selección e indicadores para la evaluación del desarrollo sostenible de un destino turístico. Aplicación al municipio de Gijón" Rotur. Revista de Ocio y Turismo, 11: 12-21, ISSN-e 2695-6357 DOI: https:// doi.org/10.17979/rotur.2016.11.1.1777 